\title{
Spin and charge currents driven by the Higgs mode in high-field superconductors
}

\author{
Mikhail A. Silaev, ${ }^{1,2}$ Risto Ojajärvi $\odot,{ }^{1}$ and Tero T. Heikkilä ${ }^{1}$ \\ ${ }^{1}$ University of Jyvaskyla, Department of Physics and Nanoscience Center, P.O. Box 35 (YFL), FI-40014 University of Jyväskylä, Finland \\ ${ }^{2}$ Moscow Institute of Physics and Technology, Dolgoprudny, Moscow Region 141700, Russia
}

(Received 1 July 2019; revised 23 August 2020; accepted 25 August 2020; published 15 September 2020)

\begin{abstract}
The Higgs mode in superconducting materials describes slowly decaying oscillations of the order parameter amplitude. We demonstrate that in superconductors with a built-in spin-splitting field the Higgs mode is strongly coupled to the spin degrees of freedom, allowing for the generation of time-dependent spin currents. Converting such spin currents to electric signals by spin-filtering elements provides a tool for the second-harmonic generation and the electrical detection of the Higgs mode generated by the external irradiation. The nonadiabatic spin torques generated by these spin currents allow for the magnetic detection of the Higgs mode by measuring the precession of the magnetic moment in the adjacent ferromagnet. We discuss also the reciprocal effect, which is the generation of the Higgs mode by the magnetic precession. Coupling the collective modes in superconductors to light and magnetic dynamics provides an opportunity for the study of superconducting optospintronics.
\end{abstract}

DOI: 10.1103/PhysRevResearch.2.033416

\section{INTRODUCTION}

Oscillations of the order parameter amplitude in condensed-matter systems are often called Higgs modes (HMs) [1-5], in analogy with the Higgs boson in particle physics [6]. These collective excitations are generic for ordered states such as antiferromagnets, charge density waves [7], superfluids [8-10], cold atomic gases [11,12] and superconductors [2,13-27]. In general, one can call HMs all the possible collective modes of the order parameter, other than the Nambu-Goldstone modes [4].

Higgs modes have been observed by Raman scattering in superconductors with charge density wave order [14,15,17,28] and by the nuclear magnetic resonance in superfluid ${ }^{3} \mathrm{He}$ [8-10]. In usual superconductors the HMs are charge neutral and thus decoupled from charge current. In such systems the observation of HMs has been facilitated by the development of low-temperature terahertz spectroscopy [18,19,24,29-32]. With this technique, HMs have been observed in NbTiN and $\mathrm{NbN}$ compounds $[18,19]$. Higgs modes have been observed indirectly as the AC linear conductance peak in currentcarrying films of $\mathrm{NbN}$ [24] and $\mathrm{Al}$ [33].

Here we suggest a different mechanism allowing electrical detection of HMs due to their coupling with spin and charge degrees of freedom in high-field superconductor/ferromagnet junctions. Unusual transport properties of such systems have attracted intense attention [34-37], stimulating both experimental [38-47] and theoretical efforts [34,48-57].

The underlying physical mechanism behind the suggested electrical measurement of the HM is rooted in the strong coupling between the superconducting order parameter dynamics

Published by the American Physical Society under the terms of the Creative Commons Attribution 4.0 International license. Further distribution of this work must maintain attribution to the author(s) and the published article's title, journal citation, and DOI. and electron spins. The possibility to transmit spin signals by the order parameter excitations has been elucidated using the example of mobile topological defects, i.e., Abrikosov vortices $[55,56]$. Here we demonstrate that time-dependent spin currents can be generated by the collective amplitude modes in superconductors.

The structure of this paper is as follows. In Sec. II we introduce the setup and model. Section III shows the effect of the HM on AC spin and charge currents. Their use in accessing the HM either in second-harmonic generation or via the measurement of an avoided crossing between ferromagnetic resonance and HMs is discussed in Sec. IV. We conclude in Sec. $\mathrm{V}$ with an outlook to the range of phenomena affected by the HM.

\section{SETUP AND MODEL}

The generic setup that we study is shown in Fig. 1(a). Its basic element is a superconducting film placed in contact with a ferromagnetic (FM) material. An effective spin-splitting field $\boldsymbol{h}$ entering as the Zeeman term in the Hamiltonian of the superconductor (SC) is induced by an external in-plane magnetic field. Alternatively, $\boldsymbol{h}$ could be induced by the proximity to a ferromagnetic insulator [58-62]. The system is exposed to an external irradiation $E_{\Omega} e^{i \Omega t}$ which generates a time-dependent perturbation of the order parameter amplitude $\delta \Delta(t)=\Delta_{2 \Omega} e^{2 i \Omega t}$ through the second-order nonlinearity $\Delta_{2 \Omega} \propto E_{\Omega}^{2}[63,64]$.

We model the SC/FM junction using the tunneling Hamiltonian approach $[65,66]$, which has been used extensively to study both AC and DC tunnel currents [65,67-69],

$$
\begin{gathered}
H_{T}=\sum_{k k^{\prime} \alpha} A_{k \alpha}^{\dagger}\left(\hat{\Gamma} \hat{B}_{k^{\prime}}\right)_{\alpha}+\text { H.c. }, \\
\hat{\Gamma}=\mathcal{T} \hat{\tau}_{3}+\mathcal{U}(\boldsymbol{m} \cdot \hat{\boldsymbol{\sigma}}) .
\end{gathered}
$$

Here $A_{k \alpha}\left(B_{k \alpha}\right)$ annihilates an electron with momentum $k$ and spin $\alpha$ in the SC (ferromagnet), the unit vector $\boldsymbol{m}$ 


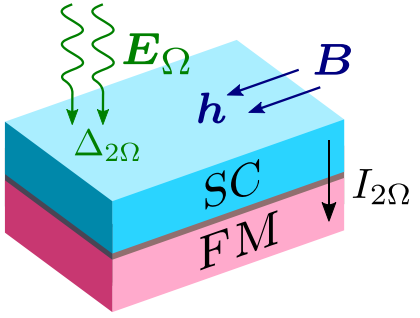

(a)

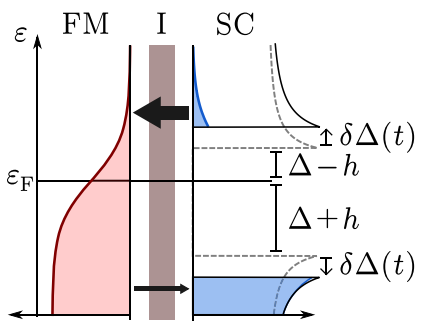

(b)
FIG. 1. (a) Setup of the superconductor/ferromagnet structure. The exchange field $\boldsymbol{h}$ is induced by an external magnetic field $\boldsymbol{B}$. The double-frequency gap modulation can be excited by the external electromagnetic irradiation $\Delta_{2 \Omega} \propto E_{\Omega}^{2}$ and is enhanced due to the coupling to the HM. (b) Semiconductor model of the current generation by a slowly varying perturbation of the order parameter amplitude $\delta \Delta(t)$. Only the spin-down band is shown. The ferromagnet has an equilibrium distribution $n_{0}(\varepsilon)$, while the distribution $n(\varepsilon, t)$ of the superconductor is shifted with respect to the equilibrium distribution (dashed line) by $\delta \Delta(t)$ away from the Fermi level. Due to the Zeeman shift $h$, the perturbation in the number of excitations is asymmetric with respect to the Fermi level $\varepsilon_{F}$. This results in net spin and charge currents flowing into the attached FM electrode through the tunnel barrier (I).

defines the spin quantization axis of the barrier, $\hat{\tau}_{k}$ and $\hat{\sigma}_{k}$ are the Pauli matrices in Nambu and spin spaces, respectively, and $\mathcal{U}$ and $\mathcal{T}$ are the spin-independent and spin-dependent matrix elements of the tunneling Hamiltonian, respectively [70]. The matrix tunneling current through the spin-polarized barriers can be expressed through momentumaveraged Green's functions (GFs) in the superconducting and FM electrodes $v_{S} \hat{g}_{S}=\hat{\tau}_{3} \sum_{k}\left\langle\mathbb{T} \hat{A}_{k}(\tau) \hat{A}_{k}^{\dagger}\left(\tau^{\prime}\right)\right\rangle$ and $v_{F} \hat{g}_{F}=$ $\hat{\tau}_{3} \sum_{k}\left\langle\mathbb{T} \hat{B}_{k}(\tau) \hat{B}_{k}^{\dagger}\left(\tau^{\prime}\right)\right\rangle$, respectively. Here $\tau$ and $\tau^{\prime}$ are imaginary times, $\mathbb{T}$ is the time-ordering operator, and $v_{S}$ and $v_{F}$ are the normal metal densities of states on the two sides of the junction. For simplicity, we assume momentum-independent tunneling coefficients [70,71]. The time-dependent tunneling current for the general nonequilibrium state in the electrodes derived in Appendix A reads

$$
\hat{I}(\tau)=i \frac{\nu_{S} \nu_{F}}{2}\left[\hat{g}_{S} \circ\left(\hat{\Gamma} \hat{g}_{F} \hat{\Gamma}\right)-\left(\hat{\Gamma} \hat{g}_{F} \hat{\Gamma}\right) \circ \hat{g}_{S}\right]_{\tau^{\prime}=\tau},
$$

where $\circ$ denotes time convolution. The overall tunnel current amplitude is determined by $\kappa=v_{S} v_{F}\left(\mathcal{T}^{2}+\mathcal{U}^{2}\right)$ and the effective spin-filtering polarization is $\boldsymbol{P}=2 \mathcal{T} \mathcal{U} \boldsymbol{m} /\left(\mathcal{T}^{2}+\mathcal{U}^{2}\right)$.

Tracing the general expression with appropriate Pauli matrices, we extract the charge current $I=e \operatorname{Tr}\left(\hat{\tau}_{3} \hat{I}\right)$ and the spin current $\boldsymbol{I}_{s}=\operatorname{Tr}(\hat{\boldsymbol{\sigma}} \hat{I})$. The real-time response is obtained by the method of analytic continuation, described in Appendix B.

We assume that the electrodes are in the diffusive regime and can be described by the time-dependent Usadel equation for quasiclassical GFs. In the imaginary-time representation it has the form (we set $\hbar=1$ here and below)

$$
-i\left\{\hat{\tau}_{3} \partial_{\tau}, \hat{g}\right\}_{\tau}=D \hat{\partial}_{\mathbf{r}}\left(\hat{g} \circ \hat{\partial}_{\mathbf{r}} \hat{g}\right)-i\left[\hat{\tau}_{3} \hat{H}, \hat{g}\right]_{\tau},
$$

where $D$ is the diffusion constant, $\hat{H}=\Delta \hat{\tau}_{1}+\boldsymbol{h} \cdot \hat{\boldsymbol{\sigma}}$, and $\boldsymbol{h}$ is the exchange field. The quasiclassical GFs also satisfy the normalization condition $(\hat{g} \circ \hat{g})_{\tau, \tau^{\prime}}=\delta\left(\tau-\tau^{\prime}\right)$. The time derivative, convolution product, and differential superoperator in Eq. (4) are

$$
\begin{gathered}
\left\{\hat{\tau}_{3} \partial_{\tau}, \hat{g}\right\}_{\tau}=\hat{\tau}_{3} \partial_{\tau_{1}} \hat{g}\left(\tau_{1}, \tau_{2}\right)+\partial_{\tau_{2}} \hat{g}\left(\tau_{1}, \tau_{2}\right) \hat{\tau}_{3}, \\
(f \circ g)\left(\tau_{1}, \tau_{2}\right)=\int_{0}^{\beta} d \tau_{3} f\left(\tau_{1}, \tau_{3}\right) g\left(\tau_{3}, \tau_{2}\right), \\
\hat{\partial}_{\mathbf{r}}=\partial_{\mathbf{r}}-\frac{i e}{c}\left[\hat{\tau}_{3} A(\tau), \cdot\right],
\end{gathered}
$$

respectively, where $e$ is the elementary charge and $c$ is the speed of light.

\section{RESULTS}

\section{A. Qualitative description}

In Fig. 1(b) we adapt the usual semiconductor picture of the tunnel current in superconductor junctions [72] to show how the time-dependent gap function creates a nonequilibrium state $n(\varepsilon, t)$ in the superconducting electrode. Due to the Zeeman shift $h$, this state is nonsymmetric with respect to the Fermi level $\varepsilon_{F}$ and therefore produces spin current through the tunnel barrier between the SC and the adjacent normal metal. This qualitative picture is based on the time-dependent energy spectrum $E_{\sigma}=\sqrt{\xi_{p}^{2}+\Delta(t)^{2}}+\sigma h$, with $\sigma= \pm 1$ for spin-up/down Bogoliubov quasiparticles, respectively, where $\xi_{p}$ is the kinetic energy counted from the Fermi level $\varepsilon_{F}$.

For a slow time-dependent order parameter the spinresolved perturbation of the quasiparticle distribution function can be written as $(\dot{\Delta} / \Gamma) \frac{d}{d \Delta} N_{\sigma}$, where the number of thermally excited states in equilibrium is $N_{\sigma}=\int d \xi_{p} n\left(E_{\sigma}\left(\xi_{p}, \Delta\right)\right)$, with $n(E)=\tanh (E / 2 T)$. The inelastic scattering relaxation rate $\Gamma$ is given by the Dynes parameter [73].

The spin-dependent perturbation of the distribution function results in the spin current

$$
I_{s}(t)=\frac{\kappa}{\Gamma} \dot{\Delta} \frac{d}{d \Delta}\left(N_{+}-N_{-}\right),
$$

where $\kappa$ is the effective barrier transparency. As shown below, Eq. (8) is obtained in the low-frequency limit $\Omega \ll \Gamma$ from the general result (21). The advantage of Eq. (8) is that it allows for the cartoon interpretation in terms of the semiconductor model in Fig. 1(b). However, for the most interesting case when the frequency of the $\Delta(t) \propto e^{2 i \Omega t}$ oscillation is comparable to the gap $\Omega \sim \Delta$ and hence is coupled to the HM [13,16,74,75], the picture becomes more complicated and requires calculations using Eqs. (3) and (4) as described in Sec. III C.

\section{B. Second-harmonic generation due to the broken particle-hole symmetry}

Spin current generated by the HM can be converted to charge current using spin-filtering FM electrodes. In the setup shown in Fig. 1(a) the spin current is effectively converted to the charge current while passing through the spin-filtering barrier characterized by the polarization vector $\boldsymbol{P}$. The time-dependent charge current induced in this way by the order parameter amplitude oscillation is therefore qualitatively given by $I(t) \propto \boldsymbol{P} \cdot \boldsymbol{I}_{s}(t)$, which results in the 
estimate $I(t) \propto(\boldsymbol{P} \cdot \boldsymbol{h}) \partial_{t} \Delta$. Modulation of the order parameter amplitude can be induced, for example, by an external irradiation [63,64] $\Delta(t) \propto \boldsymbol{A}^{2}(t)$, where $\boldsymbol{A}(t)$ is the vector potential of the external field. Hence this charge current $I(t) \propto$ $(\boldsymbol{P} \cdot \boldsymbol{h}) \partial_{t} \boldsymbol{A}^{2}$, being quadratic in the vector potential, demonstrates the second-harmonic generation (SHG) controlled by the superconducting order parameter.

Despite the large amount of attention to the nonlinear effects in superconductors, SHG has not been obtained before. ${ }^{1}$ Hence only third-harmonic generation has been studied in superconductors $[19,32,63,64,76]$. We show below that such a kind of SHG is not prohibited by the generic symmetries of the problem, but is eliminated by the approximate symmetry of Fermi surface systems, made exact in the widely used quasiclassical approximation [77]. ${ }^{2}$ This additional symmetry of the GF satisfying the Usadel equation (4) is

$$
\hat{g}(\boldsymbol{A}, \boldsymbol{h}, \Delta)=-\hat{\tau}_{1} \hat{g}\left(-\boldsymbol{A}, \boldsymbol{h}, \Delta^{*}\right) \hat{\tau}_{1} .
$$

The off-diagonal Nambu space Pauli matrix $\hat{\tau}_{1}$ interchanges the particle and hole blocks in the Hamiltonian [77], so the physical interpretation of Eq. (9) is a particle-hole symmetry. For the nonstationary charge current generated by the timedependent vector potential this symmetry yields $I(A, \Delta)=$ $-I\left(-\boldsymbol{A}, \Delta^{*}\right)$. Further, in the absence of supercurrent or external orbital fields we can assume the order parameter to be real $\Delta=\Delta^{*}$. Then even the broken inversion symmetry near surfaces does not help to produce SHG in superconducting systems in contrast to the normal metal counterpart of this effect. Because of this symmetry, the direct coupling between the HM and the charge current is prohibited. However, as we see below, it allows for the spin currents driven by the HM and external field even with a nonferromagnetic barrier, that is, at $P=0$.

The particle-hole symmetry is broken in superconducting/ FM systems leading to large thermoelectric [34,78,79] and anomalous Josephson effects [77]. For real $\Delta$ the transformation (9) applied to the general tunnel current yields

$$
I(\boldsymbol{A}, \boldsymbol{h}, \boldsymbol{P})=-I(-\boldsymbol{A}, \boldsymbol{h},-\boldsymbol{P}) .
$$

This relation allows for SHG. Because the sign of $\boldsymbol{P}$ is inverted there is no longer a symmetry with respect to the mere flipping of the vector potential, $\boldsymbol{I}(\boldsymbol{A}) \neq-\boldsymbol{I}(-\boldsymbol{A})$. Hence, for the AC external field $\boldsymbol{A}_{\Omega} e^{i \Omega t}$, Eq. (10) allows for the double-frequency charge current component $I_{2 \Omega} e^{i \Omega t}$ with the amplitude $I_{2 \Omega} \propto$ $|\Delta|^{2} A_{\Omega}^{2}(\boldsymbol{P} \cdot \boldsymbol{h})$ as well as the DC tunnel current [51] $I_{\mathrm{DC}} \propto$ $|\Delta|^{2} A_{\Omega} A_{-\Omega}(\boldsymbol{P} \cdot \boldsymbol{h})$. The resonant SHG of spin and charge currents through the excitation of HM by electromagnetic irradiation is discussed below in Sec. III C.

\section{Calculation of spin and charge currents}

We assume that the superconducting electrode is driven out of equilibrium by the electromagnetic field described by the time-dependent vector potential $A_{\Omega} e^{i \Omega t}$. It produces

\footnotetext{
${ }^{1}$ Here we exclude the trivial SHG which results from the third-order nonlinearity when both the oscillating and constant fields are applied.

${ }^{2}$ The Fermi surface symmetry present in the quasiclassical approximation is broken here by the spin polarization of tunneling.
}

the second-harmonic perturbation of the GF and tunnel current (3)

$$
\begin{gathered}
\hat{g}_{S}\left(\tau, \tau^{\prime}\right)=T \sum_{\omega} \hat{g}_{S}\left(\omega_{+}, \omega_{-}\right) e^{i \omega_{+} \tau-i \omega_{-} \tau^{\prime}}, \\
\hat{I}_{2 \Omega}=i \frac{v_{S} v_{F}}{2} T \sum_{\omega}\left[\hat{\Gamma} \hat{g}_{S} \hat{\Gamma} \hat{g}_{0}\left(\omega_{-}\right)-\hat{g}_{0}\left(\omega_{+}\right) \hat{\Gamma} \hat{g}_{S} \hat{\Gamma}\right],
\end{gathered}
$$

where $\omega_{ \pm}=\omega \pm \Omega$ are the fermionic Matsubara frequencies shifted by the frequency $\Omega$ of the external field. We define $\hat{g}_{S}=\hat{g}_{S}\left(\omega_{+}, \omega_{-}\right)$and assume that the ferromagnet is in the equilibrium state determined by the GF $\hat{g}_{F}(\omega)=\hat{g}_{0}(\omega) \equiv$ $\operatorname{sgn}(\omega) \hat{\tau}_{3}$.

There are two qualitatively different terms in the nonequilibrium GF $\hat{g}_{S}=\hat{g}_{A A}+\hat{g}_{\Delta}$. The first one is generated by the direct coupling to the external electromagnetic field. The second term is generated by the order parameter oscillations, which can be induced by either the electromagnetic field or other sources, for example, the spin current. Direct coupling to the electromagnetic field is described by the GF perturbations of second order by the vector potential.

From the Usadel equation (4) we find that the perturbation $\hat{g}_{A A}\left(\omega_{+}, \omega_{-}\right)$satisfies the equation

$$
\begin{aligned}
& s_{+} \hat{g}_{0}\left(\omega_{+}\right) \hat{g}_{A A}-s_{-} \hat{g}_{A A} \hat{g}_{0}\left(\omega_{-}\right) \\
& \quad=D\left(\frac{e A_{\Omega}}{c}\right)^{2}\left[\hat{g}_{0}\left(\omega_{+}\right) \hat{\tau}_{3} \hat{g}_{0}(\omega) \hat{\tau}_{3}-\hat{\tau}_{3} \hat{g}_{0}(\omega) \hat{\tau}_{3} \hat{g}_{0}\left(\omega_{-}\right)\right] .
\end{aligned}
$$

Expanding the normalization condition in perturbation series provides an anticommutation rule $\hat{g}_{A A} \hat{g}_{0}\left(\omega_{-}\right)=-\hat{g}_{0}\left(\omega_{+}\right) \hat{g}_{A A}$, which can be used to solve Eq. (13),

$$
\hat{g}_{A A}=D\left(\frac{e A_{\Omega}}{c}\right)^{2} \frac{\hat{\tau}_{3} \hat{g}_{0}(\omega) \hat{\tau}_{3}-\hat{g}_{0}\left(\omega_{+}\right) \hat{\tau}_{3} \hat{g}_{0}(\omega) \hat{\tau}_{3} \hat{g}_{0}\left(\omega_{-}\right)}{s_{+}+s_{-}},
$$

where $s_{ \pm}=\hat{s}\left(\omega_{ \pm}\right)$and $\hat{s}(\omega)=\sqrt{\Delta^{2}-(i \omega-\boldsymbol{h} \cdot \hat{\boldsymbol{\sigma}})^{2}}$. Above, we abuse the notation slightly by writing the matrix inverse $\left[s_{+}+s_{-}\right]^{-1}$ as a scalar division. No ambiguity is introduced as $\hat{s}(\omega)$ 's commute with the terms in the numerator. Furthermore, in the final expression for the spin current (21) we shift the energy integration and remove the exchange field from the $\hat{s}(\omega)$ 's, making their spin structure trivial.

Corrections to the GF induced by the time-dependent order parameter amplitude $\Delta_{2 \Omega} e^{2 i \Omega t}$ can be found in the form $\hat{g}_{\Delta}\left(\tau, \tau^{\prime}\right)=T \sum_{\omega} e^{i\left(\omega_{+} \tau-\omega_{-} \tau^{\prime}\right)} \hat{g}_{\Delta}\left(\omega_{+}, \omega_{-}\right)$. From the Usadel equation (4) that $\hat{g}_{\Delta}\left(\omega_{+}, \omega_{-}\right)$satisfies the equation

$$
s_{+} \hat{g}_{0}\left(\omega_{+}\right) \hat{g}_{\Delta}-s_{-} \hat{g}_{\Delta} \hat{g}_{0}\left(\omega_{-}\right)=\Delta_{2 \Omega}\left[\hat{\tau}_{2} \hat{g}_{0}\left(\omega_{-}\right)-\hat{g}_{0}\left(\omega_{+}\right) \hat{\tau}_{2}\right] \text {. }
$$

Again using the normalization condition, the solution of this equation is given by

$$
\hat{g}_{\Delta}=\Delta_{2 \Omega} \frac{\hat{g}_{0}\left(\omega_{+}\right) \hat{\tau}_{2} \hat{g}_{0}\left(\omega_{-}\right)-\hat{\tau}_{2}}{s_{+}+s_{-}} .
$$

The amplitude $\Delta_{2 \Omega}$ can be found from the self-consistency equation

$$
\Delta_{2 \Omega}=-\lambda T \sum_{\omega} \operatorname{Tr}\left[\hat{\tau}_{2}\left(\hat{g}_{A A}+\hat{g}_{\Delta}\right)\right]
$$


where we introduce the dimensionless pairing constant $\lambda$ and the Pauli matrix $\hat{\tau}_{2}$ corresponds to the superconducting amplitude vertex.

The part which is directly produced by the irradiation provides a source of the Higgs mode

$$
F_{\Delta}(2 \Omega)=-\lambda T \sum_{\omega} \operatorname{Tr}\left[\hat{\tau}_{2} \hat{g}_{A A}\right]
$$

The other part determines the self-induced corrections to the order parameter $\tilde{\Delta}_{2 \Omega}=\Pi(2 \Omega) \Delta_{2 \Omega}$ described by the polarization operator

$$
\Pi(2 \Omega)=1+\pi \lambda T \sum_{\omega} \operatorname{Tr}\left[\frac{\Delta^{2}+\Omega^{2}}{s_{-} s_{+}\left(s_{-}+s_{+}\right)}\right],
$$

where the trace is taken over the spin degree of freedom. Collecting all the contributions to the self-consistency equation (17), we get

$$
\Delta_{2 \Omega}=F_{\Delta} /[1-\Pi(2 \Omega)] .
$$

This expression describes the HM excitation in the superconductor driven out of equilibrium by a continuous-wave irradiation as shown schematically in Fig. 1(a). The resonance condition corresponding to the HM is satisfied for $\Omega=\Omega^{*}$ when $1-\Pi\left(2 \Omega^{*}\right)=0+o(\sqrt{\Gamma})$. Hence the maximal amplitude of the order parameter oscillations is determined by the broadening parameter $\Gamma$, leading to a sharp peak in $\Delta_{2 \Omega}(\Omega, T)$ for $\Omega \approx \Omega^{*}(T)$. In the absence of spin relaxation processes $\Omega^{*}=\Delta(T)$.

Using the found GF corrections (14) and (16), we calculate the spin and charge components of the tunneling current (12). The HM contribution is determined by the term $\hat{g}_{\Delta}$. Using the procedure of analytical continuation described in Appendix B, we obtain the amplitude of real-frequency spin current $\boldsymbol{I}_{s}(\Omega) e^{2 i \Omega t}$ driven by the HM,

$$
\boldsymbol{I}_{s}(\Omega)=i \kappa \boldsymbol{h} \Delta_{2 \Omega} \Delta \sum_{\sigma} \frac{\sigma}{h} \int \frac{d \varepsilon}{4 \pi} \frac{\varepsilon\left[n\left(\varepsilon_{+}\right)-n\left(\varepsilon_{-}\right)\right]}{s_{+}^{R} s_{-}^{A}\left(s_{+}^{R}+s_{-}^{A}\right)},
$$

where $n(\varepsilon)$ is the equilibrium distribution function. Here the spin splitting has been shifted from the spectral functions to the distribution functions, so $\varepsilon_{ \pm}=\varepsilon \pm \Omega+\sigma h$ and $s^{R, A}=$ $-i \sqrt{(\varepsilon \pm i \Gamma)^{2}-\Delta^{2}}$. In the low-frequency limit $\Omega \ll \Gamma$ we obtain Eq. (8) when the spin current is driven by the adiabatic time dependence of $\Delta$ in accordance with the qualitative picture shown schematically in Fig. 1(b).

In the presence of the HM, which is the slowly decaying oscillations of the order parameter $\Delta(t)[13,16]$, the spin current is given by the sum of the corresponding Fourier components with the amplitudes given by (21). As a result of Eq. (21) we get slowly decaying oscillations of the spin current $\boldsymbol{I}_{s}(t)$ which can be measured using electrical probes after the superconductor is initially driven into a nonequilibrium state by a field pulse.

Taking into account the relation (20), we obtain the SHG spin and charge currents induced by the external irradiation in accordance with the qualitative discussion in Sec. III B. The resonant behavior of the double-frequency spin current $\boldsymbol{I}_{S}(\Omega)$ resulting from the HM mode excitation is shown in Fig. 2.
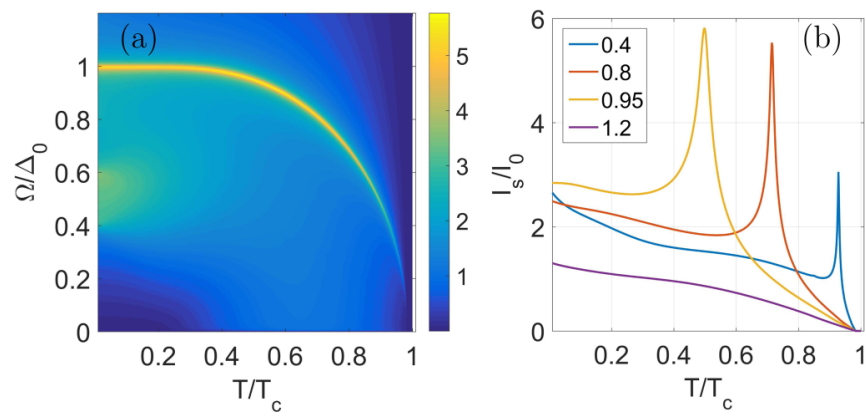

FIG. 2. Amplitude of double-frequency spin current $I_{s}(\Omega) e^{2 i \Omega t}$ driven through the $\mathrm{SC} /$ ferromagnet tunnel junction by an external field $A_{\Omega} e^{i \Omega t}$. The current is normalized to $I_{0}=\kappa D\left(e A_{\Omega} / c\right)^{2}$; $T_{c}$ is the critical temperature. The sharp maximum at frequencies $\Pi(2 \Omega) \approx 1$ corresponds to the resonant excitation of the Higgs mode. (a) $I_{s}(\Omega, T)$ and (b) $I_{s}(T)$ at different frequencies $\Omega / \Delta_{0}=$ $0.4,0.8,0.95,1.2$. The exchange field is $h=0.2 \Delta_{0}$ and the Dynes parameter $\Gamma=0.005 \Delta_{0}$. The peaks are at temperatures determined by $\Pi(2 \Omega) \approx 1$.

\section{DISCUSSION}

\section{A. Electrical detection of the Higgs mode}

The suggested effect of SHG charge current coupled to the $\mathrm{HM}$ can be measured, for example, in thin films of Al superconductor placed in a tunnel contact with FM iron electrodes similar to the setups used in the measurement of the nonlocal spin signals [38-40,43-47]. With $T_{c}=1.6 \mathrm{~K}$ and gap $\Delta_{0}=2 \times 10^{-4} \mathrm{eV}$, the spin-splitting field $h=0.2 \Delta_{0}$ can be obtained with an external in-plane magnetic field $B \approx 0.5 \mathrm{~T}$, and the polarization of this type of FM contact [44] is $P=0.2$. With large enough area, the normal-state tunnel conductance can be $e \kappa=10^{-2} \mathrm{~S}$. The electromagnetic part of the setup can be similar to the experiments on stimulated superconductivity [80,81]. The electromagnetic power is characterized by the parameter $\alpha=D\left(e A_{\Omega} / c\right)^{2}$, which can be made as large as $\alpha=$ $0.1 T_{c}$ without destroying the superconductivity [82]. For $\mathrm{Al}$ it yields $\alpha \approx 10^{-5} \mathrm{eV}$. With such parameters the charge current amplitude corresponding to Fig. 2 is $e P I_{0}=20 \mathrm{nA}$, which is two orders of magnitude larger than the nonlocal thermoelectric current measured recently in a similar setup [47]. The maximal $\mathrm{HM}$ resonance frequency in $\mathrm{Al}$ is $2 \Omega=100 \mathrm{GHz}$, which is within the capability of modern spectrum analysers. A 10 -nA current across $50 \Omega$ corresponds to the signal amplitude $-113 \mathrm{dBm}$, which means that the signal-to-noise level exceeding unity can be obtained within a 1-s measurement time with state-of-the-art high-frequency microwave spectrum analysers with a noise floor about $-120 \mathrm{dBm} / \mathrm{Hz}$.

\section{B. Spin torques generated be the Higgs mode}

If the exchange field $\boldsymbol{h}$ in the SC is noncollinear with the magnetization $\boldsymbol{m}$ in the ferromagnet, the HM generates a spin torque acting on $\boldsymbol{m}$. The generic system which can realize this configuration is shown in Fig. 3(a). Here the exchange field $\boldsymbol{h} \| \boldsymbol{m}_{0}$ is created by the ferromagnetic insulator layer with a fixed magnetic moment $\boldsymbol{m}_{0}$ [34].

The spin-transfer torque (STT) generated by the HM is shown schematically in Fig. 3(a). The polarization of the 
(a)

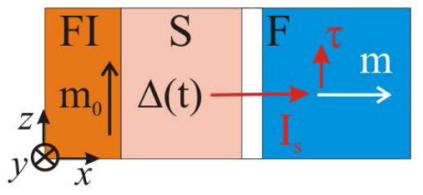

(b)

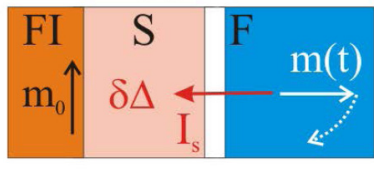

FIG. 3. Setups for studying transverse spin currents coupled to the HM in the superconductor (S). The exchange field in $\mathrm{S}$ is generated by the adjacent ferromagnetic insulator (FI) with magnetization $\boldsymbol{m}_{0}$. (a) The spin torque $\boldsymbol{\tau}$ is generated in an adjacent ferromagnet (F) with noncollinear magnetization $\boldsymbol{m} \nmid \boldsymbol{m}_{0}$. (b) Magnetization precession $\boldsymbol{m}(t)$ induces the spin current $\boldsymbol{I}_{s}$ and the spin battery effect leading to a perturbation of the order parameter amplitude $\delta \Delta$.

nonequilibrium spin current $\boldsymbol{I}_{s}$ is determined by the direction of the exchange field $\boldsymbol{h}$. Assuming that the transverse component of the spin current is absorbed in the ferromagnet [83-87], we obtain the STT $\boldsymbol{\tau}=I_{s} \boldsymbol{h}_{\perp} / h$, where $\boldsymbol{h}_{\perp}=$ $\boldsymbol{h}-\boldsymbol{m}(\boldsymbol{m} \cdot \boldsymbol{h})$ is the perpendicular component of the exchange field.

The reciprocal effect shown in Fig. 3(b) is the perturbation of the gap $\delta \Delta$ by the magnetic precession. The pumped spin current [83] $\boldsymbol{I}_{s} \propto \boldsymbol{m} \times \dot{\boldsymbol{m}}$ has a longitudinal component $\boldsymbol{I}_{s} \| \boldsymbol{h}$ which generates a time-dependent spin accumulation $\mu_{s}$ in the SC. In combination with the spin-splitting field $\boldsymbol{h}$, this results in $[51,88]$

$$
\delta \Delta=\frac{\lambda \Delta}{1-\Pi} \mu_{s} \partial_{\Delta}\left(N_{+}-N_{-}\right),
$$

where $1-\Pi \propto \lambda$ is the low-frequency asymptotic of the polarization operator. This expression demonstrates the possibility to couple the order parameter amplitude with the magnetization dynamics. Thus the higher-frequency magnetization precession with $\Omega \sim \Delta$ generates the HM in the superconductor with a spin-splitting field.

This effect can be viewed as the HM-mediated transfer of the spin angular momentum from the ferromagnetic insulator to the metallic ferromagnet shown in Fig. 3(a). Oscillating STT generated by the order parameter amplitude mode can excite the ferromagnetic resonance (FMR) in the attached ferromagnet. Hybridization of the FMR and Higgs resonance should show up as the avoided crossing of the peaks in the second-harmonic response of the systems. Such an experiment will directly demonstrate the dynamical coupling of the magnetic and superconducting orders. Modification of the FMR linewidth by superconducting correlations in ferromagnet/SC structures has been observed [89-91]. In permalloy films the FMR has been measured in fields up to $0.3 \mathrm{~T}$ corresponding to a frequency of $20 \mathrm{GHz}$. For such a frequency the Higgs resonance in $\mathrm{Al}$ is expected to occur at $T \approx 0.92 T_{c}$. Thus varying the field, one can measure the temperature-controlled hybridization of the $\mathrm{HM}$ and FMR mode in $\mathrm{Al} /$ permalloy structures within the currently accessible range of parameters.

\section{CONCLUSION}

We have demonstrated that spin and charge currents can be effectively generated by the collective amplitude modes of the superconducting order parameter. Owing to the fact that the HM can be generated by external irradiation [27,92], our result paves the way for a conceptually different direction of superconducting optospintronics: the study of spin currents and spin torques generated by light interacting with superconducting materials.

We have suggested a detection scheme for the HM based on measuring resonant electric signals, either the charge current or voltage generated across the spin-polarized tunnel junction by the external field. Because these signals appear at the doubled frequency of the external field, our setup introduces a system featuring second-harmonic generation controlled by superconductivity. The suggested SHG can be studied using optical or microwave detectors [93] and the tunneling current $I_{2 \Omega}$ can be detected using electrical probes. This feature of the SHG as compared to the previously known nonlinear response techniques allows for an electrical detection of the HM in superconductors.

Finally, a qualitatively similar effect should occur provided the ferromagnet is replaced by another spin-filtering element such as a semiconductor nanowire in proposed Majoranabased qubits [94-97]. The charge noise which is important in such devices [97] can cause the order parameter oscillations coupled to the splitting of Majorana zero modes. This coupling opens possibilities for many interesting effects to study.

\section{ACKNOWLEDGMENTS}

This work was supported by the Academy of Finland (Projects No. 297439 and No. 317118), Jenny and Antti Wihuri Foundation, Russian Science Foundation (Grant No. 19-19-00594), and the European Union's Horizon 2020 research and innovation program under Grant Agreement No. 800923 (SUPERTED).

\section{APPENDIX A: TUNNEL CURRENT}

We model the spin-dependent tunneling through the $\mathrm{SC} /$ ferromagnet interface by the tunneling Hamiltonian (1). We calculate the tunneling current as a function of the time on the contour running along the imaginary axis from 0 to $\beta=1 / T$.

The matrix tunneling current in terms of the imaginary time functions reads

$$
\hat{I}(\tau)=\frac{i}{2} \sum_{k}\left[\partial_{\tau} \hat{G}_{S}\left(\tau, \tau^{\prime}, k, k\right)+\partial_{\tau^{\prime}} \hat{G}_{S}\left(\tau, \tau^{\prime}, k, k\right)\right]_{\tau=\tau^{\prime}} .
$$

To find the perturbation we consider the contour-ordered GF

$$
\hat{G}_{S}\left(\tau_{1}, \tau_{2}, k, k^{\prime}\right)=\left\langle\mathbb{T} \hat{S} \hat{A}_{k}\left(\tau_{1}\right) \hat{A}_{k^{\prime}}^{\dagger}\left(\tau_{2}\right)\right\rangle,
$$

where $\mathbb{T}$ is the contour-ordering operator and

$$
\hat{S} \approx 1-\int_{0}^{\beta} d \tau_{3} H_{T}\left(\tau_{3}\right)
$$


In the interaction representation with respect to the tunneling Hamiltonian the equation of motion is

$$
\partial_{\tau} \hat{A}_{k}=\left[\hat{A}_{k}, H_{T}\right]=\sum_{k^{\prime}}\left(\hat{\Gamma}_{k k^{\prime}} \hat{B}_{k^{\prime}}\right)
$$

Using the equation of motion we get

$$
\begin{aligned}
-\partial_{\tau_{1}} \hat{G}_{S}\left(\tau_{1}, \tau_{2}, \boldsymbol{k}, \boldsymbol{k}\right) & =-\sum_{q}\left\langle\mathbb{T} \hat{S}\left[\hat{\Gamma}_{k q} \hat{B}_{q}\left(\tau_{1}\right)\right] \hat{A}_{k}^{\dagger}\left(\tau_{2}\right)\right\rangle \\
& \approx \sum_{q}\left\langle\mathbb{T} \int_{0}^{\beta} d \tau_{3} H_{T}\left(\tau_{3}\right) \hat{\Gamma}_{k q} \hat{B}_{q}\left(\tau_{1}\right) \hat{A}_{k}^{\dagger}\left(\tau_{2}\right)\right\rangle \\
& =\sum_{k_{1}, k_{1}^{\prime}, q}\left\langle\mathbb{T} \int_{0}^{\beta} d \tau_{3} \hat{B}_{k_{1}^{\prime}}^{\dagger}\left(\tau_{3}\right) \hat{\Gamma}_{k_{1} k_{1}^{\prime}}^{\dagger} \hat{A}_{k_{1}}\left(\tau_{3}\right) \hat{\Gamma}_{k q} \hat{B}_{q}\left(\tau_{1}\right) \hat{A}_{k}^{\dagger}\left(\tau_{2}\right)\right\rangle \\
& =\sum_{k_{1}, k_{1}^{\prime}, q} \int_{0}^{\beta} d \tau_{3} \hat{\Gamma}_{k q}\left\langle\mathbb{T} \hat{B}_{q}\left(\tau_{1}\right) \hat{B}_{k_{1}^{\prime}}^{\dagger}\left(\tau_{3}\right)\right| \hat{\Gamma}_{k_{1}^{\prime} k_{1}}\left\langle\mathbb{T} \hat{A}_{k_{1}}\left(\tau_{3}\right) \hat{A}_{k}^{\dagger}\left(\tau_{2}\right)\right\rangle \\
& =\sum_{k_{1}, k_{1}^{\prime}, q} \int_{0}^{\beta} d \tau_{3} \hat{\Gamma}_{k q} \hat{G}_{F}\left(\tau_{1}, \tau_{3}, \boldsymbol{q}, \boldsymbol{k}_{1}^{\prime}\right) \hat{\Gamma}_{k_{1}^{\prime} k_{1}} \hat{G}_{S}\left(\tau_{3}, \tau_{2}, \boldsymbol{k}_{1}, \boldsymbol{k}\right)
\end{aligned}
$$

and

$$
\begin{aligned}
-\partial_{\tau_{2}} \hat{G}_{S}\left(\tau_{1}, \tau_{2}, \boldsymbol{k}, \boldsymbol{k}\right) & =-\sum_{q}\left\langle\mathbb{T} \hat{S} \hat{A}_{k}(\tau)\left[\hat{\Gamma}_{k q} \hat{B}_{q}\left(t^{\prime}\right)\right]^{\dagger}\right\rangle \\
& \approx \sum_{q}\left\langle\mathbb{T} \int_{0}^{\beta} d \tau_{3} H_{T}\left(\tau_{3}\right) \hat{A}_{k}\left(\tau_{1}\right)\left[\hat{\Gamma}_{k q} \hat{B}_{q}\left(\tau_{2}\right)\right]^{\dagger}\right\rangle \\
& =\sum_{k_{1}, k_{1}^{\prime}, q}\left\langle\mathbb{T} \int_{0}^{\beta} d \tau_{3} \hat{\Gamma}_{k_{1} k_{1}^{\prime}} \hat{B}_{k_{1}^{\prime}}\left(\tau_{3}\right) \hat{A}_{k_{1}}^{\dagger}\left(\tau_{3}\right) \hat{A}_{k}\left(\tau_{1}\right) \hat{B}_{q}^{\dagger}\left(\tau_{2}\right) \hat{\Gamma}_{q k}^{\dagger}\right\rangle \\
& =-\sum_{k_{1}, k_{1}^{\prime}, q} \int_{0}^{\beta} d \tau_{3}\left\langle\mathbb{T} \hat{A}_{k}\left(\tau_{1}\right) \hat{A}_{k_{1}}^{\dagger}\left(\tau_{3}\right)\right\rangle \hat{\Gamma}_{k_{1} k_{1}^{\prime}}\left\langle\mathbb{T} \hat{B}_{k_{1}^{\prime}}\left(\tau_{3}\right) \hat{B}_{q}^{\dagger}\left(\tau_{2}\right)\right\rangle \hat{\Gamma}_{q k} \\
& =-\sum_{k_{1}, k_{1}^{\prime}, q} \int_{0}^{\beta} d \tau_{3} \hat{G}_{S}\left(\tau_{1}, \tau_{3}, \boldsymbol{k}, \boldsymbol{k}_{1}\right) \hat{\Gamma}_{k_{1} k_{1}^{\prime}} \hat{G}_{F}\left(\tau_{3}, \tau_{2}, \boldsymbol{k}_{1}^{\prime}, \boldsymbol{q}\right) \hat{\Gamma}_{q k} .
\end{aligned}
$$

Hence the matrix current is given by

$$
\begin{aligned}
\hat{I}(\tau)= & \frac{i}{2} \sum_{k, k_{1}, k_{1}^{\prime}, q} \int_{0}^{\beta} d \tau^{\prime}\left[\hat{G}_{S}\left(\tau, \tau^{\prime}, \boldsymbol{k}, \boldsymbol{k}_{1}\right) \hat{\Gamma}_{k_{1} k_{1}^{\prime}} \hat{G}_{F}\left(\tau^{\prime}, \tau, \boldsymbol{k}_{1}^{\prime}, \boldsymbol{q}\right) \hat{\Gamma}_{q k}\right. \\
& \left.-\hat{\Gamma}_{k q} \hat{G}_{F}\left(\tau, \tau^{\prime}, \boldsymbol{q}, \boldsymbol{k}_{1}\right) \hat{\Gamma}_{k_{1} k_{1}^{\prime}} \hat{G}_{S}\left(\tau^{\prime}, \tau, \boldsymbol{k}_{1}^{\prime}, \boldsymbol{k}\right)\right] .
\end{aligned}
$$

We assume that GFs are spatially homogeneous, so $\hat{G}_{F}\left(\tau, \tau^{\prime}, \boldsymbol{q}, \boldsymbol{k}_{1}\right)=\delta_{q, k_{1}} \hat{G}_{F}\left(\tau, \tau^{\prime}, \boldsymbol{q}\right)$ and the matrix element is momentum independent $\hat{\Gamma}_{k k^{\prime}}=\hat{\Gamma}$. Then we can introduce the quasiclassical functions $\sum_{q} \hat{G}_{F, S}\left(\tau, \tau^{\prime}, \boldsymbol{q}\right)=v_{F, S} \hat{\tau}_{3} \hat{g}_{F, S}\left(\tau, \tau^{\prime}\right)$ to write the current as

$$
\hat{I}(\tau)=i \frac{v_{S} v_{F}}{2}\left[\hat{g}_{S} \circ\left(\hat{\tau}_{3} \hat{\Gamma} \hat{g}_{F} \hat{\Gamma} \hat{\tau}_{3}\right)-\left(\hat{\tau}_{3} \hat{\Gamma} \hat{g}_{F} \hat{\Gamma} \hat{\tau}_{3}\right) \circ \hat{g}_{S}\right]_{\tau^{\prime}=\tau}
$$

Taking into account that the normal metal GF $\hat{g}_{F}$ commutes with $\hat{\tau}_{3}$, Eq. (A8) can be reduced to Eq. (3).

\section{APPENDIX B: ANALYTICAL CONTINUATION}

In order to find the real-frequency response we need to implement the analytic continuation of Eq. (12). These second-order responses are obtained by the summation of expressions which depend on the multiple shifted fermionic frequencies such as $g\left(\omega_{1}, \omega_{2}, \omega_{3}\right)$. The analytic continuation of the sum by Matsubara frequencies is determined according to the general rule [98]

$$
\begin{aligned}
T \sum_{\omega} g\left(\omega_{1}, \omega_{2}, \omega_{3}\right) \rightarrow & \sum_{l=1}^{3} \int \frac{d \varepsilon}{4 \pi i} n_{0}\left(\varepsilon_{l}\right)\left[g\left(\ldots,-i \varepsilon_{l}^{R}, \ldots\right)\right. \\
& \left.-g\left(\ldots,-i \varepsilon_{l}^{A}, \ldots\right)\right],
\end{aligned}
$$


where $n_{0}(\varepsilon)=\tanh (\varepsilon / 2 T)$ is the equilibrium distribution function. On the right-hand side of (B1) we substitute in each term $\omega_{k<l}=-i \varepsilon_{k}^{R}$ and $\omega_{k>l}=-i \varepsilon_{k}^{A}$ for $k=1,2,3$, and we define $\varepsilon_{k}=\varepsilon+(2-k) \Omega, \varepsilon^{R}=\varepsilon+i \Gamma$, and $\varepsilon^{A}=\varepsilon-i \Gamma$. Here the term with $\Gamma>0$ is added to shift the integration contour into the corresponding half plane. At the same time, $\Gamma$ can be used as the Dynes parameter [73] to describe the effect of different depairing mechanisms on spectral functions in the superconductor.

We implement the analytical continuation in such a way that $s\left(-i \varepsilon^{R, A}\right)=-i \sqrt{\left(\varepsilon^{R, A}\right)^{2}-\Delta^{2}}$, assuming that the branch cuts run from $(\Delta, \infty)$ and $(-\infty,-\Delta)$. In the presence of the spin-splitting field the energy in Eq. (B1) should be shifted to $\varepsilon+\sigma h$, where $\sigma= \pm 1$ is the spin subband index.

The equilibrium GF in the imaginary frequency domain is given by $\hat{g}_{0}(\omega)=\left(\hat{\tau}_{3} \omega-\hat{\tau}_{2} \Delta\right) / s(\omega)$. The real-frequency continuation reads $\hat{g}_{0}^{R, A}(\varepsilon)=\left(\hat{\tau}_{3} \varepsilon_{R, A}-i \hat{\tau}_{2} \Delta\right) / \sqrt{\left(\varepsilon^{R, A}\right)^{2}-\Delta^{2}}$.

Example. To demonstrate the analytical continuation in practice we calculate the spin current driven by the Higgs mode. For real frequencies the spin current obtained from (A8) can be written in terms of the Keldysh component

$$
\begin{aligned}
I_{S} & =\frac{\kappa}{8 \pi} \sum_{\sigma} \sigma \int d \varepsilon \operatorname{Tr}\left[\hat{g}_{F}\left(\varepsilon_{+}\right) \hat{g}_{S}(\varepsilon)-\hat{g}_{S}(\varepsilon) \hat{g}_{F}\left(\varepsilon_{-}\right)\right]^{K} \\
& =\frac{\kappa}{8 \pi} \sum_{\sigma} \sigma \int d \varepsilon\left[n\left(\varepsilon_{+}\right)-n\left(\varepsilon_{-}\right)\right] \operatorname{Tr}\left[\hat{\tau}_{3} \hat{g}_{S}^{a}\right],
\end{aligned}
$$

where $\varepsilon_{ \pm}=\varepsilon+\sigma h \pm \omega$. In deriving (B2) we used the fact that $\hat{g}_{F}^{R(A)}= \pm 1$ do not depend on energy. The anomalous part of the nonequilibrium GF in the superconductor is

$$
\hat{g}_{S}^{a}=\Delta_{2 \Omega} \frac{\hat{g}^{R}\left(\varepsilon_{+}\right) \hat{\tau}_{2} \hat{g}^{A}\left(\varepsilon_{-}\right)-\hat{\tau}_{2}}{s_{+}^{R}+s_{-}^{A}},
$$

where we define $s_{ \pm}^{R, A}=s^{R, A}\left(\varepsilon_{ \pm}\right)$. Substituting the solution (B3) and using $\operatorname{Tr}\left[\tau_{3} g_{+}^{R} \tau_{2} g_{-}^{A}\right]=2 i \Delta_{0} \varepsilon / s_{+}^{R} s_{-}^{A}$, we get

$$
\begin{aligned}
I_{s} & =i \kappa \Delta_{0} \Delta_{2 \Omega} \sum_{\sigma} \sigma \int \frac{d \varepsilon}{4 \pi} \frac{(\varepsilon+\sigma h)\left[n\left(\varepsilon_{+}\right)-n\left(\varepsilon_{-}\right)\right]}{s_{+}^{R} s_{-}^{A}\left(s_{+}^{R}+s_{-}^{A}\right)} \\
& =\frac{i \kappa \Delta_{0} \Delta_{2 \Omega}}{(\omega+i \Gamma)} \int \frac{d \varepsilon}{16 \pi} \sum_{\sigma} \sigma\left[n\left(\varepsilon_{+}\right)-n\left(\varepsilon_{-}\right)\right]\left(\frac{1}{s_{+}^{R}}-\frac{1}{s_{-}^{A}}\right),
\end{aligned}
$$

where we use $\left(s_{+}^{R}\right)^{2}-\left(s_{-}^{A}\right)^{2}=4(\varepsilon+\sigma h)(\omega+i \Gamma)$. In the low-frequency limit we can substitute $n\left(\varepsilon_{+}\right)-n\left(\varepsilon_{-}\right)=$ $2 \omega \partial_{\varepsilon} n$ and $s_{+}^{R}=-s_{+}^{A}=-i \sqrt{\varepsilon^{2}-\Delta^{2}}$. Then the spin current can be written in the simple form

$$
\begin{aligned}
I_{s} & =\frac{\kappa}{\Gamma} \sum_{\sigma} \sigma \frac{d}{d t} \int d \xi_{p} n\left(E_{\sigma}\left(\xi_{p}, \Delta(t)\right)\right) \\
& =\frac{\kappa}{\Gamma} \dot{\Delta} \frac{d}{d \Delta}\left(N_{+}-N_{-}\right),
\end{aligned}
$$

where $E_{\sigma}\left(\xi_{p}, \Delta(t)\right)=\sqrt{\xi_{p}^{2}+\Delta^{2}(t)}+\sigma h$ is the spectrum of Bogoliubov quasiparticles shifted by the spin-splitting field $h$.
[1] C. M. Varma, Higgs boson in superconductors, J. Low Temp. Phys. 126, 901 (2002).

[2] D. Podolsky, A. Auerbach, and D. P. Arovas, Visibility of the amplitude (Higgs) mode in condensed matter, Phys. Rev. B 84, 174522 (2011).

[3] A. Pashkin and A. Leitenstorfer, Particle physics in a superconductor, Science 345, 1121 (2014).

[4] G. E. Volovik and M. A. Zubkov, Higgs bosons in particle physics and in condensed matter, J. Low Temp. Phys. 175, 486 (2014).

[5] D. Pekker and C. M. Varma, Amplitude/Higgs modes in condensed matter physics, Annu. Rev. Condens. Matter Phys. 6, 269 (2015).

[6] P. W. Higgs, Broken Symmetries and the Masses of Gauge Bosons, Phys. Rev. Lett. 13, 508 (1964).

[7] G. Grüner, The dynamics of charge-density waves, Rev. Mod. Phys. 60, 1129 (1988).

[8] D. N. Paulson, R. T. Johnson, and J. C. Wheatley, Propagation of Collisionless Sound in Normal and Extraordinary Phases of Liquid ${ }^{3}$ He below 3 mK, Phys. Rev. Lett. 30, 829 (1973).

[9] D. T. Lawson, W. J. Gully, S. Goldstein, R. C. Richardson, and D. M. Lee, Attenuation of Zero Sound and the LowTemperature Transitions in Liquid ${ }^{3} \mathrm{He}$, Phys. Rev. Lett. 30, 541 (1973).

[10] V. V. Zavjalov, S. Autti, V. B. Eltsov, P. J. Heikkinen, and G. E. Volovik, Light Higgs channel of the resonant decay of magnon condensate in superfluid ${ }^{3} \mathrm{He}-\mathrm{B}$, Nat. Commun. 7, 10294 (2016).
[11] U. Bissbort, S. Götze, Y. Li, J. Heinze, J. S. Krauser, M. Weinberg, C. Becker, K. Sengstock, and W. Hofstetter, Detecting the Amplitude Mode of Strongly Interacting Lattice Bosons by Bragg Scattering, Phys. Rev. Lett. 106, 205303 (2011).

[12] M. Endres, T. Fukuhara, D. Pekker, M. Cheneau, P. Schauß, C. Gross, E. Demler, S. Kuhr, and I. Bloch, The 'Higgs' amplitude mode at the two-dimensional superfluid/Mott insulator transition, Nature (London) 487, 454 (2012).

[13] A. F. Volkov and S. M. Kogan, Collisionless relaxation of the energy gap in superconductors, Zh. Eksp. Teor. Fiz. 65, 2038 (1973) [Sov. Phys._JETP 38, 1018 (1974)].

[14] R. Sooryakumar and M. V. Klein, Raman Scattering by Superconducting-Gap Excitations and their Coupling to Charge-Density Waves, Phys. Rev. Lett. 45, 660 (1980).

[15] P. B. Littlewood and C. M. Varma, Amplitude collective modes in superconductors and their coupling to charge-density waves, Phys. Rev. B 26, 4883 (1982).

[16] R. A. Barankov, L. S. Levitov, and B. Z. Spivak, Collective Rabi Oscillations and Solitons in a Time-Dependent BCS Pairing Problem, Phys. Rev. Lett. 93, 160401 (2004).

[17] R. Grasset, T. Cea, Y. Gallais, M. Cazayous, A. Sacuto, L. Cario, L. Benfatto, and M.-A. Méasson, Higgs-mode radiance and charge-density-wave order in $2 \mathrm{H}-\mathrm{NbSe}_{2}$, Phys. Rev. B 97, 094502 (2018).

[18] R. Matsunaga, Y. I. Hamada, K. Makise, Y. Uzawa, H. Terai, Z. Wang, and R. Shimano, Higgs Amplitude Mode in the BCS Superconductors $\mathrm{Nb}_{1-x} \mathrm{Ti}_{x} \mathrm{~N}$ Induced by Terahertz Pulse Excitation, Phys. Rev. Lett. 111, 057002 (2013). 
[19] R. Matsunaga, N. Tsuji, H. Fujita, A. Sugioka, K. Makise, Y. Uzawa, H. Terai, Z. Wang, H. Aoki, and R. Shimano, Lightinduced collective pseudospin precession resonating with Higgs mode in a superconductor, Science 345, 1145 (2014).

[20] D. Sherman, U. S. Pracht, B. Gorshunov, S. Poran, J. Jesudasan, M. Chand, P. Raychaudhuri, M. Swanson, N. Trivedi, A. Auerbach, M. Scheffler, A. Frydman, and M. Dressel, The Higgs mode in disordered superconductors close to a quantum phase transition, Nat. Phys. 11, 188 (2015).

[21] N. Tsuji and H. Aoki, Theory of Anderson pseudospin resonance with Higgs mode in superconductors, Phys. Rev. B 92, 064508 (2015).

[22] R. Matsunaga, N. Tsuji, K. Makise, H. Terai, H. Aoki, and R. Shimano, Polarization-resolved terahertz third-harmonic generation in a single-crystal superconductor $\mathrm{NbN}$ : Dominance of the Higgs mode beyond the BCS approximation, Phys. Rev. B 96, 020505(R) (2017).

[23] K. Katsumi, N. Tsuji, Y. I. Hamada, R. Matsunaga, J. Schneeloch, R. D. Zhong, G. D. Gu, H. Aoki, Y. Gallais, and R. Shimano, Higgs Mode in the $d$-Wave Superconductor $\mathrm{Bi}_{2} \mathrm{Sr}_{2} \mathrm{CaCu}_{2} \mathrm{O}_{8+x}$ Driven by an Intense Terahertz Pulse, Phys. Rev. Lett. 120, 117001 (2018).

[24] S. Nakamura, Y. Iida, Y. Murotani, R. Matsunaga, H. Terai, and R. Shimano, Infrared Activation of the Higgs Mode by Supercurrent Injection in Superconducting NbN, Phys. Rev. Lett. 122, 257001 (2019).

[25] H. Uematsu, T. Mizushima, A. Tsuruta, S. Fujimoto, and J. A. Sauls, Chiral Higgs Mode in Nematic Superconductors, Phys. Rev. Lett. 123, 237001 (2019).

[26] A. Moor, A. F. Volkov, and K. B. Efetov, Amplitude Higgs Mode and Admittance in Superconductors with a Moving Condensate, Phys. Rev. Lett. 118, 047001 (2017).

[27] M. Silaev, Nonlinear electromagnetic response and Higgs-mode excitation in BCS superconductors with impurities, Phys. Rev. B 99, 224511 (2019).

[28] R. Grasset, Y. Gallais, A. Sacuto, M. Cazayous, S. MañasValero, E. Coronado, and M.-A. Méasson, Pressure-Induced Collapse of the Charge Density Wave and Higgs Mode Visibility in $2 \mathrm{H}-\mathrm{TaS}_{2}$, Phys. Rev. Lett. 122, 127001 (2019).

[29] M. Beck, M. Klammer, S. Lang, P. Leiderer, V. V. Kabanov, G. N. Gol'tsman, and J. Demsar, Energy-Gap Dynamics of Superconducting NbN Thin Films Studied by TimeResolved Terahertz Spectroscopy, Phys. Rev. Lett. 107, 177007 (2011).

[30] M. Beck, I. Rousseau, M. Klammer, P. Leiderer, M. Mittendorff, S. Winnerl, M. Helm, G. N. Gol'tsman, and J. Demsar, Transient Increase of the Energy Gap of Superconducting NbN Thin Films Excited by Resonant Narrow-Band Terahertz Pulses, Phys. Rev. Lett. 110, 267003 (2013).

[31] R. Matsunaga and R. Shimano, Nonequilibrium BCS State Dynamics Induced by Intense Terahertz Pulses in a Superconducting NbN Film, Phys. Rev. Lett. 109, 187002 (2012).

[32] F. Giorgianni, T. Cea, C. Vicario, C. P. Hauri, W. K. Withanage, $\mathrm{X}$. Xi, and L. Benfatto, Leggett mode controlled by light pulses, Nat. Phys. 15, 341 (2019).

[33] W. V. Budzinski, M. P. Garfunkel, and R. W. Markley, Magnetic field dependence of the surface resistance of pure and impure superconducting aluminum at photon energies near the energy gap, Phys. Rev. B 7, 1001 (1973).
[34] F. S. Bergeret, M. Silaev, P. Virtanen, and T. T. Heikkilä, Colloquium: Nonequilibrium effects in superconductors with a spin-splitting field, Rev. Mod. Phys. 90, 041001 (2018).

[35] T. T. Heikkilä, M. Silaev, P. Virtanen, and F. S. Bergeret, Thermal, electric and spin transport in superconductor/ferromagnetic-insulator structures, Prog. Surf. Sci. 94, 100540 (2019).

[36] D. Beckmann, Spin manipulation in nanoscale superconductors, J. Phys.: Condens. Matter 28, 163001 (2016).

[37] C. H. L. Quay and M. Aprili, Out-of-equilibrium spin transport in mesoscopic superconductors, Philos. Trans. R. Soc. A 376, 20150342 (2018).

[38] F. Hübler, M. J. Wolf, D. Beckmann, and H. v. Löhneysen, Long-Range Spin-Polarized Quasiparticle Transport in Mesoscopic Al Superconductors with a Zeeman Splitting, Phys. Rev. Lett. 109, 207001 (2012).

[39] M. J. Wolf, C. Sürgers, G. Fischer, and D. Beckmann, Spin-polarized quasiparticle transport in exchange-split superconducting aluminum on europium sulfide, Phys. Rev. B 90, 144509 (2014).

[40] M. Rouco, S. Chakraborty, F. Aikebaier, V. N. Golovach, E. Strambini, J. S. Moodera, F. Giazotto, T. T. Heikkilä, and F. S. Bergeret, Charge transport through spin-polarized tunnel junction between two spin-split superconductors, Phys. Rev. B 100, 184501 (2019).

[41] G. De Simoni, E. Strambini, J. S. Moodera, F. S. Bergeret, and F. Giazotto, Toward the absolute spin-valve effect in superconducting tunnel junctions, Nano Lett. 18, 6369 (2018).

[42] E. Strambini, V. N. Golovach, G. De Simoni, J. S. Moodera, F. S. Bergeret, and F. Giazotto, Revealing the magnetic proximity effect in EuS/Al bilayers through superconducting tunneling spectroscopy, Phys. Rev. Mater. 1, 054402 (2017).

[43] S. Kolenda, M. J. Wolf, and D. Beckmann, Observation of Thermoelectric Currents in High-Field SuperconductorFerromagnet Tunnel Junctions, Phys. Rev. Lett. 116, 097001 (2016).

[44] M. J. Wolf, F. Hübler, S. Kolenda, H. v. Löhneysen, and D. Beckmann, Spin injection from a normal metal into a mesoscopic superconductor, Phys. Rev. B 87, 024517 (2013).

[45] C. H. L. Quay, D. Chevallier, C. Bena, and M. Aprili, Spin imbalance and spin-charge separation in a mesoscopic superconductor, Nat. Phys. 9, 84 (2013).

[46] C. H. L. Quay, C. Dutreix, D. Chevallier, C. Bena, and M. Aprili, Frequency-domain measurement of the spin-imbalance lifetime in superconductors, Phys. Rev. B 93, 220501(R) (2016).

[47] J. Heidrich and D. Beckmann, Nonlocal thermoelectric effects in high-field superconductor-ferromagnet hybrid structures, Phys. Rev. B 100, 134501 (2019).

[48] M. Silaev, P. Virtanen, F. S. Bergeret, and T. T. Heikkilä, LongRange Spin Accumulation from Heat Injection in Mesoscopic Superconductors with Zeeman Splitting, Phys. Rev. Lett. 114, 167002 (2015).

[49] I. V. Bobkova and A. M. Bobkov, Long-range spin imbalance in mesoscopic superconductors under Zeeman splitting, JETP Lett. 101, 118 (2015).

[50] T. Krishtop, M. Houzet, and J. S. Meyer, Nonequilibrium spin transport in Zeeman-split superconductors, Phys. Rev. B 91, 121407(R) (2015). 
[51] P. Virtanen, T. T. Heikkilä, and F. S. Bergeret, Stimulated quasiparticles in spin-split superconductors, Phys. Rev. B 93, 014512 (2016).

[52] F. Aikebaier, M. A. Silaev, and T. T. Heikkilä, Supercurrentinduced charge-spin conversion in spin-split superconductors, Phys. Rev. B 98, 024516 (2018).

[53] P. Virtanen, F. S. Bergeret, E. Strambini, F. Giazotto, and A. Braggio, Majorana bound states in hybrid two-dimensional Josephson junctions with ferromagnetic insulators, Phys. Rev. B 98, 020501(R) (2018).

[54] I. V. Bobkova and A. M. Bobkov, Injection of nonequilibrium quasiparticles into Zeeman-split superconductors: A way to create long-range spin imbalance, Phys. Rev. B 93, 024513 (2016).

[55] S. K. Kim, R. Myers, and Y. Tserkovnyak, Nonlocal Spin Transport Mediated by a Vortex Liquid in Superconductors, Phys. Rev. Lett. 121, 187203 (2018).

[56] A. Vargunin and M. Silaev, Flux flow spin Hall effect in typeII superconductors with spin-splitting field, Sci. Rep. 9, 5914 (2019).

[57] V. L. Vadimov, I. M. Khaymovich, and A. S. Mel'nikov, Higgs modes in proximized superconducting systems, Phys. Rev. B 100, 104515 (2019).

[58] F. S. Bergeret, A. F. Volkov, and K. B. Efetov, Enhancement of the Josephson Current by an Exchange Field in SuperconductorFerromagnet Structures, Phys. Rev. Lett. 86, 3140 (2001).

[59] T. Tokuyasu, J. A. Sauls, and D. Rainer, Proximity effect of a ferromagnetic insulator in contact with a superconductor, Phys. Rev. B 38, 8823 (1988).

[60] A. Millis, D. Rainer, and J. A. Sauls, Quasiclassical theory of superconductivity near magnetically active interfaces, Phys. Rev. B 38, 4504 (1988).

[61] A. Cottet, D. Huertas-Hernando, W. Belzig, and Y. V. Nazarov, Spin-dependent boundary conditions for isotropic superconducting Green's functions, Phys. Rev. B 80, 184511 (2009).

[62] M. Eschrig, A. Cottet, W. Belzig, and J. Linder, General boundary conditions for quasiclassical theory of superconductivity in the diffusive limit: Application to strongly spin-polarized systems, New J. Phys. 17, 083037 (2015).

[63] L. P. Gor'kov and G. M. Éliashberg, Superconducting alloys in a strong alternating field, Zh. Eksp. Teor. Fiz. 56, 1297 (1969) [Sov. Phys._JETP 29, 698 (1969)].

[64] L. P. Gor'kov and G. M. Éliashberg, The behavior of a superconductor in a variable field, Zh. Eksp. Teor. Fiz. 55, 2430 (1968) [Sov. Phys._JETP 28, 1291 (1969)].

[65] V. Ambegaokar and A. Baratoff, Tunneling between Superconductors, Phys. Rev. Lett. 10, 486 (1963).

[66] J. Bardeen, Tunneling into Superconductors, Phys. Rev. Lett. 9 , 147 (1962).

[67] U. Eckern, G. Schön, and V. Ambegaokar, Quantum dynamics of a superconducting tunnel junction, Phys. Rev. B 30, 6419 (1984).

[68] R. E. Harris, Josephson tunneling current in the presence of a time-dependent voltage, Phys. Rev. B 11, 3329 (1975).

[69] N. R. Werthamer, Nonlinear self-coupling of Josephson radiation in superconducting tunnel junctions, Phys. Rev. 147, 255 (1966).

[70] F. S. Bergeret, A. Verso, and A. F. Volkov, Electronic transport through ferromagnetic and superconducting junctions with spin-filter tunneling barriers, Phys. Rev. B 86, 214516 (2012).
[71] F. S. Bergeret, A. Verso, and A. F. Volkov, Spin-polarized Josephson and quasiparticle currents in superconducting spin-filter tunnel junctions, Phys. Rev. B 86, 060506(R) (2012).

[72] M. Tinkham, Introduction to Superconductivity (Courier, Chelmsford, 2004).

[73] R. C. Dynes, J. P. Garno, G. B. Hertel, and T. P. Orlando, Tunneling Study of Superconductivity near the Metal-Insulator Transition, Phys. Rev. Lett. 53, 2437 (1984).

[74] I. O. Kulik, O. Entin-Wohlman, and R. Orbach, Pair susceptibility and mode propagation in superconductors: A microscopic approach, J. Low Temp. Phys. 43, 591 (1981).

[75] R. A. Barankov and L. S. Levitov, Synchronization in the BCS Pairing Dynamics as a Critical Phenomenon, Phys. Rev. Lett. 96, 230403 (2006).

[76] J. C. Amato and W. L. McLean, Measurement of the Superconducting Order-Parameter Relaxation Time from Harmonic Generation, Phys. Rev. Lett. 37, 930 (1976).

[77] M. A. Silaev, I. V. Tokatly, and F. S. Bergeret, Anomalous current in diffusive ferromagnetic Josephson junctions, Phys. Rev. B 95, 184508 (2017).

[78] P. Machon, M. Eschrig, and W. Belzig, Nonlocal Thermoelectric Effects and Nonlocal Onsager Relations in a ThreeTerminal Proximity-Coupled Superconductor-Ferromagnet Device, Phys. Rev. Lett. 110, 047002 (2013).

[79] A. Ozaeta, P. Virtanen, F. S. Bergeret, and T. T. Heikkilä, Predicted Very Large Thermoelectric Effect in FerromagnetSuperconductor Junctions in the Presence of a Spin-Splitting Magnetic Field, Phys. Rev. Lett. 112, 057001 (2014).

[80] T. M. Klapwijk, J. N. Van Den Bergh, and J. E. Mooij, Radiation-stimulated superconductivity, J. Low Temp. Phys. 26, 385 (1977).

[81] T. Kommers and J. Clarke, Measurement of MicrowaveEnhanced Energy Gap in Superconducting Aluminum by Tunneling, Phys. Rev. Lett. 38, 1091 (1977).

[82] K. S. Tikhonov, M. A. Skvortsov, and T. M. Klapwijk, Superconductivity in the presence of microwaves: Full phase diagram, Phys. Rev. B 97, 184516 (2018).

[83] Y. Tserkovnyak, A. Brataas, G. E. W. Bauer, and B. I. Halperin, Nonlocal magnetization dynamics in ferromagnetic heterostructures, Rev. Mod. Phys. 77, 1375 (2005).

[84] J. C. Slonczewski, Current-driven excitation of magnetic multilayers, J. Magn. Magn. Mater. 159, L1 (1996).

[85] A. Brataas, Y. V. Nazarov, and G. E. W. Bauer, Finite-Element Theory of Transport in Ferromagnet-Normal Metal Systems, Phys. Rev. Lett. 84, 2481 (2000).

[86] X. Waintal, E. B. Myers, P. W. Brouwer, and D. C. Ralph, Role of spin-dependent interface scattering in generating currentinduced torques in magnetic multilayers, Phys. Rev. B 62 , 12317 (2000).

[87] M. D. Stiles and A. Zangwill, Anatomy of spin-transfer torque, Phys. Rev. B 66, 014407 (2002).

[88] I. V. Bobkova and A. M. Bobkov, Thermospin effects in superconducting heterostructures, Phys. Rev. B 96, 104515 (2017).

[89] C. Bell, S. Milikisyants, M. Huber, and J. Aarts, Spin Dynamics in a Superconductor-Ferromagnet Proximity System, Phys. Rev. Lett. 100, 047002 (2008).

[90] K.-R. Jeon, C. Ciccarelli, A. J. Ferguson, H. Kurebayashi, L. F. Cohen, X. Montiel, M. Eschrig, J. W. A. Robinson, and M. G. Blamire, Enhanced spin pumping into superconductors 
provides evidence for superconducting pure spin currents, Nat. Mater. 17, 499 (2018).

[91] K.-R. Jeon, C. Ciccarelli, H. Kurebayashi, L. F. Cohen, X. Montiel, M. Eschrig, S. Komori, J. W. A. Robinson, and M. G. Blamire, Exchange-field enhancement of superconducting spin pumping, Phys. Rev. B 99, 024507 (2019).

[92] Y. Murotani and R. Shimano, Nonlinear optical response of collective modes in multiband superconductors assisted by nonmagnetic impurities, Phys. Rev. B 99, 224510 (2019).

[93] T. T. Heikkilä, R. Ojajärvi, I. J. Maasilta, E. Strambini, F. Giazotto, and F. S. Bergeret, Thermoelectric Radiation Detector Based on Superconductor-Ferromagnet Systems, Phys. Rev. Appl. 10, 034053 (2018).

[94] T. Hyart, B. van Heck, I. C. Fulga, M. Burrello, A. R. Akhmerov, and C. W. J. Beenakker, Flux-controlled quantum computation with Majorana fermions, Phys. Rev. B 88, 035121 (2013).

[95] T. Karzig, C. Knapp, R. M. Lutchyn, P. Bonderson, M. B. Hastings, C. Nayak, J. Alicea, K. Flensberg, S. Plugge, Y. Oreg, C. M. Marcus, and M. H. Freedman, Scalable designs for quasiparticle-poisoning-protected topological quantum computation with Majorana zero modes, Phys. Rev. B 95, 235305 (2017).

[96] S. Plugge, A. Rasmussen, R. Egger, and K. Flensberg, Majorana box qubits, New J. Phys. 19, 012001 (2017).

[97] C. Knapp, T. Karzig, R. M. Lutchyn, and C. Nayak, Dephasing of Majorana-based qubits, Phys. Rev. B 97, 125404 (2018).

[98] N. B. Kopnin, Theory of Nonequilibrium Superconductivity (Oxford University Press, Oxford, 2001). 